\title{
Efecto de la suplementación con harina de mashua (Tropaeolum tuberosum) y tarwi (Lupinus mutabilis) sobre la respuesta productiva y composición nutricional de cuyes (Cavia porcellus)
}

\author{
Effect of the supplementation of mashua (Tropaeolum tuberosum) and tarwi \\ (Lupinus mutabilis) meal on the productive response and nutritional \\ composition of guinea pigs (Cavia porcellus)
}

\author{
Sayuri Rosangela Acuña-Beraunn ${ }^{1,3}$, Nancy Párraga-Melgarejo ${ }^{1}$, \\ Daniel Alvarez-Tolentino ${ }^{2}$
}

\section{Resumen}

\begin{abstract}
Se evaluó el efecto de la suplementación con harina de mashua (Tropaeolum tuberosum) y tarwi (Lupinus mutabilis) sobre la ganancia de peso vivo (GPV), consumo de alimento, índice de conversión alimenticia y composición nutricional de carcasas de cuyes de la raza Perú. Se utilizaron 32 cuyes machos de 4 semanas de edad (309-578 g) distribuidos aleatoriamente en cuatro tratamientos $(\mathrm{T})$ durante 40 días: $\mathrm{T} 1$ (concentrado integral - CI), T2 (CI $+18 \%$ tarwi), T3 (CI $+25 \%$ mashua) y T4 $(\mathrm{CI}+25 \%$ mashua $+18 \%$ tarwi). La suplementación con harina de tarwi (T2 y T4) mejoró la GPV, en tanto que la suplementación con harina de mashua (T3) tuvo un efecto significativo en el contenido de grasa de la carcasa $(\mathrm{p}<0.05)$. No hubo efecto en el consumo de alimentos y conversión alimenticia.
\end{abstract}

Palabras clave: suplementación, indicadores de productividad, valor nutricional, Cavia porcellus, tarwi, mashua

\section{AbStract}

The effect of supplementation with mashua (Tropaeolum tuberosum) and tarwi (Lupinus mutabilis) meal on body weight gain (BWG), feed consumption, feed conversion index and nutritional composition of carcasses of Peru breed guinea pigs was evaluated.

\footnotetext{
${ }^{1}$ Escuela de Ingeniería Agroindustrial, Universidad Nacional del Centro del Perú (UNCP), Tarma, Junín, Perú

${ }^{2}$ Instituto General de Investigación (IGINV), Universidad Nacional del Centro del Perú (UNCP), Huancayo, Junín, Perú

${ }^{3}$ E-mail: sayuacuna@gmail.com
}

Recibido: 10 de agosto de 2020

Aceptado para publicación; 21 de enero de 2021

Publicado: 24 de abril de 2021 
Peru. For the study, 32 4-week-old male guinea pigs (309-578 g) were randomly distributed in four treatments ( $\mathrm{T}$ ) for 40 days: T1 (integral concentrate - IC), T2 (CI + 18\% tarwi), T3 (IC $+25 \%$ mashua) and T4 (I C $+25 \%$ mashua $+18 \%$ tarwi). Supplementation with tarwi meal (T2 and T4) improved BWG, while supplementation with mashua meal (T3) had a significant effect on carcass fat content $(\mathrm{p}<0.05)$. There was no effect on feed consumption and feed conversion.

Key words: supplementation, productivity indicators, nutritional value, Cavia porcellus, tarwi, mashua

\section{INTRODUCCIÓN}

En el Perú se viene incrementando la producción y consumo de carne de cuy (Sánchez, 2019; Cuibin et al., 2020). Las últimas cifras indican que el volumen de exportación en 2018 fue de $9958 \mathrm{~kg}$ y el valor de mercado en las fronteras aduaneras (FOB, por sus siglas en inglés) fue 128,000 dólares (MINAGRI, 2019). Se estima una población de 18 millones de cuyes en el país, distribuidos en 188 productores grandes y 17192 productores medianos o pequeños (INEI, 2018).

A pesar del crecimiento productivo, la mayoría de los productores realiza la crianza con un manejo tradicional, ocasionando sobrecostos y menor calidad productiva (Zambrano, 2015; Salvatierra et al., 2018). Un punto crítico es la alimentación no balanceada (Tineo, 2015; Collado, 2016; Sotelo et al., 2020). Ante esto, diversos estudios han evaluado suplementos nutricionales para la optimización de la producción de cuyes, obteniendo buenos resultados (Quintana et al., 2013; Rosales et al., 2014; León et al., 2016; Reyes-Sánchez et al., 2018; Sullca, 2019; Hidalgo y Valerio, 2020).

En la parte andina de la región central del Perú (Junín), existe gran producción agrícola de mashua (Tropaeolum tuberosum) y tarwi (Lupinus mutabilis) (MINAGRI, 2018); no obstante, a pesar de que pueden reemplazar a la soya (Ojeda y Salazar, 2011) y al maíz (Urresta, 2010) por su alto valor nutritivo (INS, 2017), son poco valorados para su uso en las raciones alimenticias. Con el fin de aprovechar las propiedades nutritivas del tarwi y mashua, el presente estudio tuvo por objetivo evaluar el efecto de la suplementación de estos nutrientes sobre los indicadores productivos y valor nutricional de los cuyes de la sierra central. Esto permitirá nuevas posibilidades de alimentación en la producción de cuyes.

\section{Materiales y Métodod}

\section{Ubicación}

La investigación se llevó a cabo entre julio a setiembre de 2019 en las instalaciones de la granja de cuyes de la Escuela de Ingeniería Agroindustrial de la Universidad Nacional del Centro del Perú (UNCP), ubicada en la ciudad de Tarma, departamento de Junín, Perú. La zona presenta una temperatura media anual de $12{ }^{\circ} \mathrm{C}$ y una precipitación de $459 \mathrm{~mm}$.

\section{Material Biológico}

Se emplearon 32 cuyes machos destetados de raza Perú con cuatro semanas de edad, pesos de 309 a $578 \mathrm{~g}$, distribuidos aleatoriamente en cuatro jaulas de acero galvanizado y $73.5 \mathrm{~cm}^{3}$ de espacio. Para minimizar el sesgo, se colocaron pesos homogéneos de cuyes en cada jaula evaluados con la prueba de Levene $(\mathrm{p}>0.05)$. 
Cuadro 1. Análisis químico proximal de las dietas experimentales evaluadas (en base fresca)

\begin{tabular}{lcccc}
\hline Componentes & \multicolumn{4}{c}{ Tratamientos } \\
\cline { 2 - 5 }$(\mathrm{g} / 100 \mathrm{~g})$ & $\mathrm{T} 1$ & $\mathrm{~T} 2$ & $\mathrm{~T} 3$ & $\mathrm{~T} 4$ \\
\hline Materia seca & 96.8 & 99.23 & 98.02 & 97.42 \\
Proteína & 12.84 & 18.28 & 12.21 & 24.71 \\
Fibra cruda & 31.03 & 18.81 & 16.47 & 17.37 \\
Grasa & 6.23 & 9.20 & 11.25 & 9.17 \\
Cenizas & 7.88 & 7.47 & 9.91 & 8.05 \\
Extracto libre N & 38.82 & 45.47 & 48.18 & 38.12 \\
\hline
\end{tabular}

T1: concentrado integral (control); T2: concentrado integral $+18 \%$ harina de tarwi; T3: concentrado integral $+25 \%$ harina de mashua; T4: concentrado integral $+25 \%$ harina de mashua $+18 \%$ harina de tarwi.

Fuente: Laboratorio de la Escuela de Ingeniería Agroindustrial - UNCP

\section{Diseño Experimental}

Se utilizó un diseño completamente aleatorio (DCA) durante 40 días. En cada jaula se ubicó a un grupo de 8 cuyes (repeticiones) formando cuatro tratamientos de alimentación: T1: concentrado integral (control); T2: concentrado integral $+18 \%$ harina de tarwi; T3: concentrado integral $+25 \%$ harina de mashua; T4: concentrado integral $+25 \%$ harina de mashua $+18 \%$ harina de tarwi.

\section{Dietas}

Se preparó un concentrado integral según las recomendaciones del Consejo Nacional de Investigación (NRC, 1995), donde se adicionó las harinas de tarwi y mashua. Las mezclas según los tratamientos fueron realizadas con una balanza electrónica de $0.1 \mathrm{~g}$ de sensibilidad.

La harina de tarwi fue obtenida después de la limpieza de los granos con hipoclorito- de sodio (150 ppm por 5 minutos), hidratación (1:2 grano/agua por 12 horas), cocción (60 minutos), desamargado (lavado 6 veces al día), secado ( $80^{\circ} \mathrm{C}$ por 24 horas). La harina de mashua fue obtenida después de la limpieza de los tubérculos con hipoclorito de sodio (200 ppm por 5 minutos), rectificado en rodajas (2 mm espesor), secado $\left(60^{\circ} \mathrm{C}\right.$ por 24 horas). La molienda en ambos se hizo en un molino de martillos.

Diariamente se suministró $100 \mathrm{~g}$ de alimento, tipo concentrado peletizado con diámetro de $4 \mathrm{~mm}$ y longitud de $12 \mathrm{~mm}$ por cada animal en comederos de arcilla enlozada. La mitad del alimento a las 07:00 y el resto a las 19:00. Se suministró agua ad libitum en bebederos de plástico.

Se tomaron $500 \mathrm{~g}$ de muestras de las dietas experimentales al inicio de la etapa para el análisis químico proximal en el Laboratorio de la Escuela de Ingeniería Agroindustrial de la UNCP (Cuadro 1). 
Cuadro 2. Métodos de análisis para la composición nutricional en carcasas de cuyes de la raza Perú

\begin{tabular}{|c|c|}
\hline Parámetro & Método \\
\hline Humedad & $\begin{array}{l}\text { Método } 930.15 \text { (AOAC, 1990) en una estufa Memmert a } 100^{\circ} \mathrm{C} \\
\text { durante } 6 \mathrm{~h} \text {. }\end{array}$ \\
\hline Grasa & $\begin{array}{l}\text { Método Soxhlet (AOAC, 1990) utilizando como disolvente al } \\
\text { hexano } 250 \mathrm{ml} \text { en un balón de } 500 \mathrm{ml} \text { por cada análisis realizado. }\end{array}$ \\
\hline Proteína & $\begin{array}{l}\text { Método } 976.05 \text { Kjeldahl automatizado (AOAC, 1990). Se utilizó el } \\
\text { Sistema de Destilación Vapodest }{ }^{\circledR}-\text { Gerhardt y Bloque de Digestión } \\
\text { Kjeldatherm }{ }^{\circledR} \text { - Gerhardt. }\end{array}$ \\
\hline Ceniza & $\begin{array}{l}\text { Método } 942.05 \text { (AOAC, 1990). Se pesó } 1 \mathrm{~g} \text { de muestra en crisol y se } \\
\text { llevó a la mufla a } 600^{\circ} \mathrm{C} \text { durante } 6 \mathrm{~h} \text {. }\end{array}$ \\
\hline Fibra & $\begin{array}{l}\text { Método } 962.09 \text { (AOAC, 1990). Se utilizó } 5 \mathrm{~g} \text { de muestra que se llevó } \\
\text { a ebullición con } 250 \mathrm{ml} \text { de ácido sulfúrico } 1.25 \% \text { durante } 30 \text { min y } \\
\text { de la misma manera luego con el hidróxido de sodio } 1.25 \% \text {. La } \\
\text { muestra es puesta en placa Petri para ser secada en estufa a } 100{ }^{\circ} \mathrm{C} \\
\text { durante } 6 \text { h y por último en la mufla a } 600{ }^{\circ} \mathrm{C} \text { durante } 3 \mathrm{~h} \text {. }\end{array}$ \\
\hline $\begin{array}{l}\text { Extracto libre } \\
\text { de Nitrógeno }\end{array}$ & Se realizó por diferencia con el total. \\
\hline
\end{tabular}

\section{Variables}

- Ganancia de peso vivo (GPV). Se registraron los pesos individuales de cada animal desde el inicio del experimento y cada siete días. Se empleó una balanza electrónica de $0.1 \mathrm{~g}$ de sensibilidad con el cuy en ayunas (Salcedo, 2017).

- Consumo de alimento (CA). Diariamente se pesó el residuo no consumido. La CA fue calculada por diferencia entre los pesos del alimento ofrecido y no consumido.

- Índice de conversión alimenticia (ICA). Se calculó dividiendo el CA y la GPV en cada unidad experimental (Salcedo, 2017).

- Composición nutricional (CN). Se determinó el contenido de humedad, grasa, proteína, ceniza, fibra y extracto libre de nitrógeno de las carcasas al final del experimento, según las recomendaciones de la Asociación Oficial de Químicos Analistas (AOAC, 1990). El detalle de los métodos se muestra en el Cuadro 2.

\section{Análisis Estadístico}

Se calcularon los promedios y desviación estándar de la GPV, CA e ICA por semana y tratamiento. Se aplicó el test de Saphiro-Wilk para determinar la normalidad de los datos y la prueba de Kruskal-Wallis para la comparación de los tratamientos $(\alpha=$ 0.05 ). Además, se aplicó la prueba de MannWhitney para comparaciones entre tratamientos. Para los análisis y pruebas se utilizó el software estadístico MINITAB v. 18. 


\section{Resultados}

\section{Indicadores Productivos}

Ganancia de peso vivo

En el Cuadro 3 se aprecia el comportamiento de la GPV de los cuyes según la dieta ofrecida durante las seis semanas del estudio. Los tratamientos mostraron diferencias significativas $(\mathrm{p}<0.05)$, hasta la quinta semana, siendo mayor en T4 (concentrado integral + $25 \%$ harina de mashua $+18 \%$ harina de tarwi); sin embargo, no hubo diferencias significativas entre tratamientos en la semana 6.

Los promedios de GPV por tratamiento se muestran en la Figura 1. Los cuyes de T4 presentaron la mayor ganancia $(239.46 \pm$ $77.65 \mathrm{~g} / \mathrm{semana})$, seguida del T2. La menor ganancia se observó en los cuyes de T3 (concentrado integral $+25 \%$ de harina de mashua). Los resultados muestran un mejor efecto con los suplementos con tarwi (T2 y T4) en el incremento de peso $(\mathrm{p}<0.05)$.

Consumo de alimento

El CA semanal no mostró diferencia significativa entre tratamientos durante las seis semanas del estudio (Cuadro 4).
En la Figura 2 se puede observar los promedios de CA toda la duración del experimento, donde los cuyes de T4 (485.92 \pm 90.82 $\mathrm{g} /$ semana) y T2 (430.50 $\pm 74.48 \mathrm{~g} /$ semana $)$ presentaron mayores consumos, a pesar de no mostrar diferencia significativa entre tratamientos $(\mathrm{p}<0.05)$.

\section{Índice de conversión alimenticia}

La primera y segunda semana se registraron conversiones alimenticias bajas sin diferencia significativa, y los grupos suplementados con mashua mostraron bajas conversiones en la tercera semana $(\mathrm{p}<0.05$; Cuadro 5).

La Figura 3 muestra los promedios de conversión alimenticia durante el estudio. Los mejores promedios se obtuvieron en los cuyes de los tratamientos suplementados con tarwi (T2 y T4), aunque sin diferencia significativa.

\section{Composición Nutricional}

El Cuadro 6 muestra los componentes nutricionales de las carcasas de cuy según las dietas suministradas. Se encontró un mayor contenido de grasa en las carcasas de cuyes de $\mathrm{T} 3(\mathrm{p}<0.05)$ y mayor contenido de fibra en T1 $(p<0.05)$. De otro lado, hubo di-

Cuadro 3. Ganancia de peso vivo (promedio $\pm \mathrm{DE}$ ) semanal de cuyes de la raza Perú según tratamientos (Tarma, Perú)

\begin{tabular}{ccccccc}
\hline & Semana 1 & Semana 2 & Semana 3 & Semana 4 & Semana 5 & Semana 6 \\
\hline T1 & $10.4 \pm 16.8^{\mathrm{b}}$ & $62.6 \pm 43.5^{\mathrm{c}}$ & $178.2 \pm 87.5^{\mathrm{b}}$ & $248.1 \pm 103.4^{\mathrm{ab}}$ & $288.5 \pm 93.5^{\mathrm{ab}}$ & $317.6 \pm 89.6^{\mathrm{a}}$ \\
$\mathrm{T} 2$ & $23.5 \pm 16.2^{\mathrm{ab}}$ & $171.4 \pm 71.0^{\mathrm{a}}$ & $225.1 \pm 82.3^{\mathrm{ab}}$ & $289.9 \pm 92.9^{\mathrm{a}}$ & $301.6 \pm 93.5^{\mathrm{ab}}$ & $341.2 \pm 94.5^{\mathrm{a}}$ \\
$\mathrm{T} 3$ & $21.8 \pm 13.2^{\mathrm{ab}}$ & $75.1 \pm 41.1^{\mathrm{bc}}$ & $117.5 \pm 47.1^{\mathrm{b}}$ & $159.2 \pm 49.3^{\mathrm{b}}$ & $204.1 \pm 53.0^{\mathrm{b}}$ & $268.2 \pm 36.7^{\mathrm{a}}$ \\
$\mathrm{T} 4$ & $33.6 \pm 14.3^{\mathrm{a}}$ & $162.8 \pm 92.8^{\mathrm{ab}}$ & $239.5 \pm 96.2^{\mathrm{a}}$ & $308.2 \pm 93.1^{\mathrm{a}}$ & $322.6 \pm 90.4^{\mathrm{a}}$ & $370.0 \pm 90.7^{\mathrm{a}}$ \\
\hline$p$ & 0.04 & 0.003 & 0.022 & 0.009 & 0.045 & 0.106 \\
\hline
\end{tabular}

T1: concentrado integral (control); T2: concentrado integral $+18 \%$ harina de tarwi; T3: concentrado integral $+25 \%$ harina de mashua; $\mathrm{T} 4$ : concentrado integral $+25 \%$ harina de mashua $+18 \%$ harina de tarwi.

$\mathrm{a}, \mathrm{b}, \mathrm{c}$ Valores con superíndices diferentes dentro de columnas indican diferencias significativas $(p<0.05)$ 
Cuadro 4. Consumo de alimento (promedio \pm DE) semanal de cuyes de la raza Perú según tratamientos (Tarma, Perú)

\begin{tabular}{ccccccc}
\hline & Semana 1 & Semana 2 & Semana 3 & Semana 4 & Semana 5 & Semana 6 \\
\hline T1 & $430.5 \pm 24.8^{\mathrm{a}}$ & $392.0 \pm 79.2^{\mathrm{a}}$ & $420.0 \pm 188.1^{\mathrm{a}}$ & $315.0 \pm 29.7^{\mathrm{a}}$ & $427.0 \pm 49.5^{\mathrm{a}}$ & $427.0 \pm 108.9^{\mathrm{a}}$ \\
T2 & $525.0 \pm 59.4^{\mathrm{a}}$ & $385.0 \pm 49.5^{\mathrm{a}}$ & $518.0 \pm 89.1^{\mathrm{a}}$ & $395.5 \pm 193.0^{\mathrm{a}}$ & $343.0 \pm 128.7^{\mathrm{a}}$ & $416.5 \pm 94.0^{\mathrm{a}}$ \\
T3 & $364.0 \pm 79.2^{\mathrm{a}}$ & $427.0 \pm 9.9^{\mathrm{a}}$ & $560.0 \pm 49.5^{\mathrm{a}}$ & $273.0 \pm 19.8^{\mathrm{a}}$ & $357.0 \pm 108.9^{\mathrm{a}}$ & $420.0 \pm 99.0^{\mathrm{a}}$ \\
T4 & $511.0 \pm 49.5^{\mathrm{a}}$ & $437.5 \pm 44.6^{\mathrm{a}}$ & $616.0 \pm 89.1^{\mathrm{a}}$ & $535.5 \pm 64.4^{\mathrm{a}}$ & $350.0 \pm 198.0^{\mathrm{a}}$ & $465.5 \pm 163.3^{\mathrm{a}}$ \\
\hline$p$ & 0.129 & 0.707 & 0.472 & 0.198 & 0.909 & 0.972 \\
\hline
\end{tabular}

T1: concentrado integral (control); T2: concentrado integral $+18 \%$ harina de tarwi; T3: concentrado integral $+25 \%$ harina de mashua; $\mathrm{T} 4$ : concentrado integral $+25 \%$ harina de mashua $+18 \%$ harina de tarwi.

${ }^{a}$ Valores con superíndices similares no son significativamente diferentes $(p>0.05)$

Cuadro 5. Conversión alimenticia (promedio \pm DE) semanal de cuyes de la raza Perú según tratamientos (Tarma, Perú)

\begin{tabular}{ccccccc}
\hline & Semana 1 & Semana 2 & Semana 3 & Semana 4 & Semana 5 & Semana 6 \\
\hline T1 & $3.60 \pm 6.34^{\mathrm{a}}$ & $2.83 \pm 1.94^{\mathrm{a}}$ & $2.26 \pm 0.76^{\mathrm{b}}$ & $1.46 \pm 0.56^{\mathrm{a}}$ & $1.69 \pm 0.66^{\mathrm{a}}$ & $2.31 \pm 1.09^{\mathrm{a}}$ \\
T2 & $4.55 \pm 4.66^{\mathrm{a}}$ & $2.13 \pm 1.43^{\mathrm{a}}$ & $2.09 \pm 1.16^{\mathrm{b}}$ & $1.51 \pm 0.78^{\mathrm{a}}$ & $1.11 \pm 0.46^{\mathrm{ab}}$ & $2.16 \pm 1.19^{\mathrm{a}}$ \\
T3 & $5.67 \pm 1.90^{\mathrm{a}}$ & $4.03 \pm 1.21^{\mathrm{a}}$ & $3.75 \pm 0.88^{\mathrm{a}}$ & $1.40 \pm 0.30^{\mathrm{a}}$ & $1.35 \pm 0.16^{\mathrm{ab}}$ & $3.12 \pm 0.63^{\mathrm{a}}$ \\
T4 & $4.17 \pm 2.61^{\mathrm{a}}$ & $2.12 \pm 0.99^{\mathrm{a}}$ & $2.18 \pm 0.76^{\mathrm{b}}$ & $1.79 \pm 0.59^{\mathrm{a}}$ & $1.00 \pm 0.28^{\mathrm{b}}$ & $2.14 \pm 0.78^{\mathrm{a}}$ \\
\hline$p$ & 0.096 & 0.054 & 0.003 & 0.344 & 0.019 & 0.153 \\
\hline
\end{tabular}

T1: concentrado integral (control); T2: concentrado integral $+18 \%$ harina de tarwi; T3: concentrado integral $+25 \%$ harina de mashua; T4: concentrado integral $+25 \%$ harina de mashua $+18 \%$ harina de tarwi.

$a, b$ Valores con superíndices diferentes dentro de columnas indican diferencias significativas $(\mathrm{p}<0.05)$

ferencias significativas en los contenidos de materia seca, ceniza, humedad, proteínas y extracto libre de nitrógeno entre tratamientos.

\section{Discusión}

La suplementación con harina de tarwi (T2 y T4) tuvo mejor efecto en la GPV com parada con suplementos de harina de mashua (T3) e integral (T1). Asimismo, las GPV fue mejor que la reportada en otros estudios con alimento balanceado (Solorzano et al., 2010), harina de yacón (Perea et al., 2007) y harina de sangre (Salcedo, 2017), posiblemente debido al mayor contenido proteico del tarwi (Chauca et al., 2004; INS, 2017). El consumo de alimento (CA) fue ligeramente supe- 


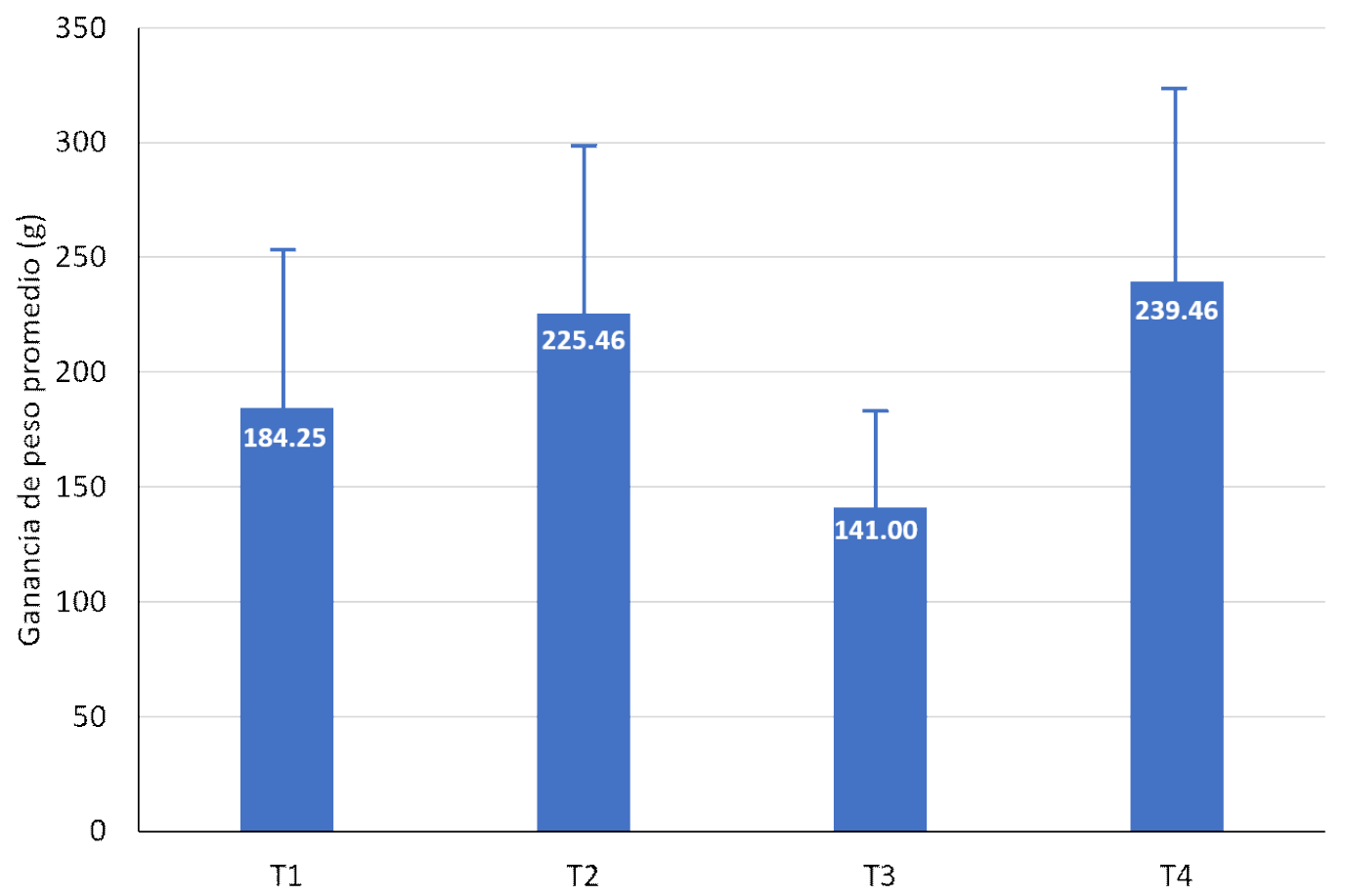

Figura 1. Promedio general de la ganancia de peso vivo (g) de cuyes de la raza Perú durante 40 días de recibir las dietas experimentales. T1: concentrado integral (control); T2: concentrado integral $+18 \%$ harina de tarwi; $T 3$ : concentrado integral $+25 \%$ harina de mashua; T4: concentrado integral $+25 \%$ harina de mashua $+18 \%$ harina de tarwi

Cuadro 6. Composición nutricional de la carcasa de cuyes de raza Perú según dietas suministradas durante 40 días

\begin{tabular}{lcccc}
\hline Componentes & \multicolumn{4}{c}{ Tratamientos } \\
\cline { 2 - 5 }$(\mathrm{g} / 100 \mathrm{~g})$ & $\mathrm{T} 1$ & $\mathrm{~T} 2$ & $\mathrm{~T} 3$ & $\mathrm{~T} 4$ \\
\hline Materia seca & $28.92 \pm 0.5^{\mathrm{a}}$ & $28.92 \pm 0.5^{\mathrm{a}}$ & $28.84 \pm 0.42^{\mathrm{a}}$ & $28.94 \pm 0.14^{\mathrm{a}}$ \\
Proteína & $8.30 \pm 0.66^{\mathrm{a}}$ & $7.81 \pm 0.65^{\mathrm{a}}$ & $8.29 \pm 1.28^{\mathrm{a}}$ & $8.99 \pm 3.85^{\mathrm{a}}$ \\
Fibra cruda & $3.91 \pm 0.13^{\mathrm{a}}$ & $2.73 \pm 0.22^{\mathrm{b}}$ & $1.48 \pm 0.21^{\mathrm{b}}$ & $1.19 \pm 0.27^{\mathrm{b}}$ \\
Grasa & $7.66 \pm 2.25^{\mathrm{b}}$ & $11.63 \pm 2.11^{\mathrm{ab}}$ & $14.32 \pm 1.40^{\mathrm{a}}$ & $11.59 \pm 2.35^{\mathrm{ab}}$ \\
Cenizas & $0.99 \pm 0.03^{\mathrm{a}}$ & $0.66 \pm 0.50^{\mathrm{a}}$ & $0.93 \pm 0.08^{\mathrm{a}}$ & $0.98 \pm 0.14^{\mathrm{a}}$ \\
Extracto libre N & $8.06 \pm 1.83^{\mathrm{a}}$ & $6.09 \pm 2.75^{\mathrm{a}}$ & $3.83 \pm 2.63^{\mathrm{a}}$ & $6.19 \pm 5.25^{\mathrm{a}}$ \\
\hline
\end{tabular}

T1: concentrado integral (control); T2: concentrado integral $+18 \%$ harina de tarwi; T3: concentrado integral $+25 \%$ harina de mashua; T4: concentrado integral $+25 \%$ harina de mashua $+18 \%$ harina de tarwi.

a,b Valores con superíndices diferentes dentro de filas indican diferencias significativas $(p<0.05)$ 


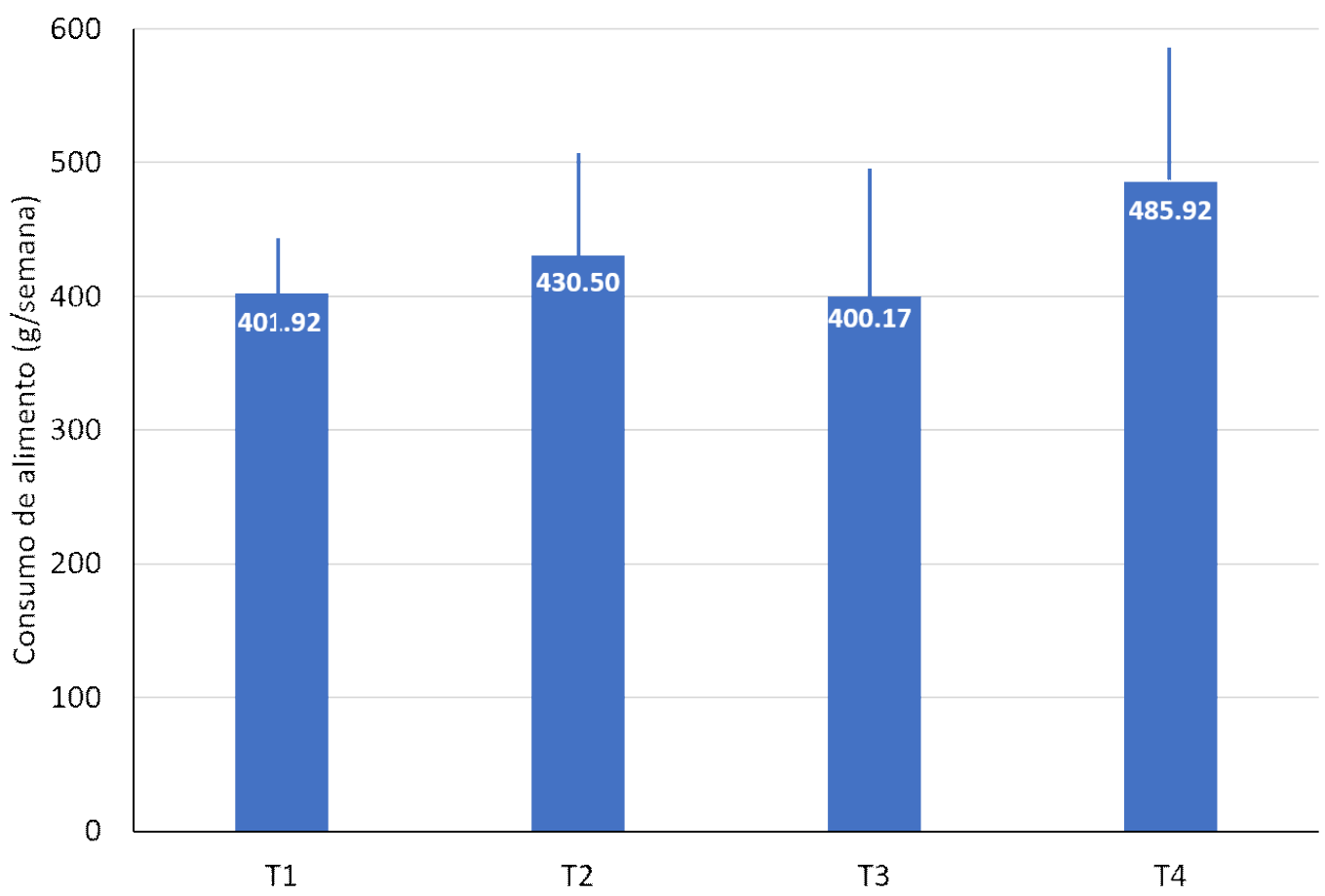

Figura 2. Consumo de alimento ( $\mathrm{g} / \mathrm{semana})$ de cuyes de la raza Perú durante 40 días de recibir las dietas experimentales. T1: concentrado integral (control); T2: concentrado integral $+18 \%$ harina de tarwi; T3: concentrado integral $+25 \%$ harina de mashua; T4: concentrado integral $+25 \%$ harina de mashua $+18 \%$ harina de tarwi

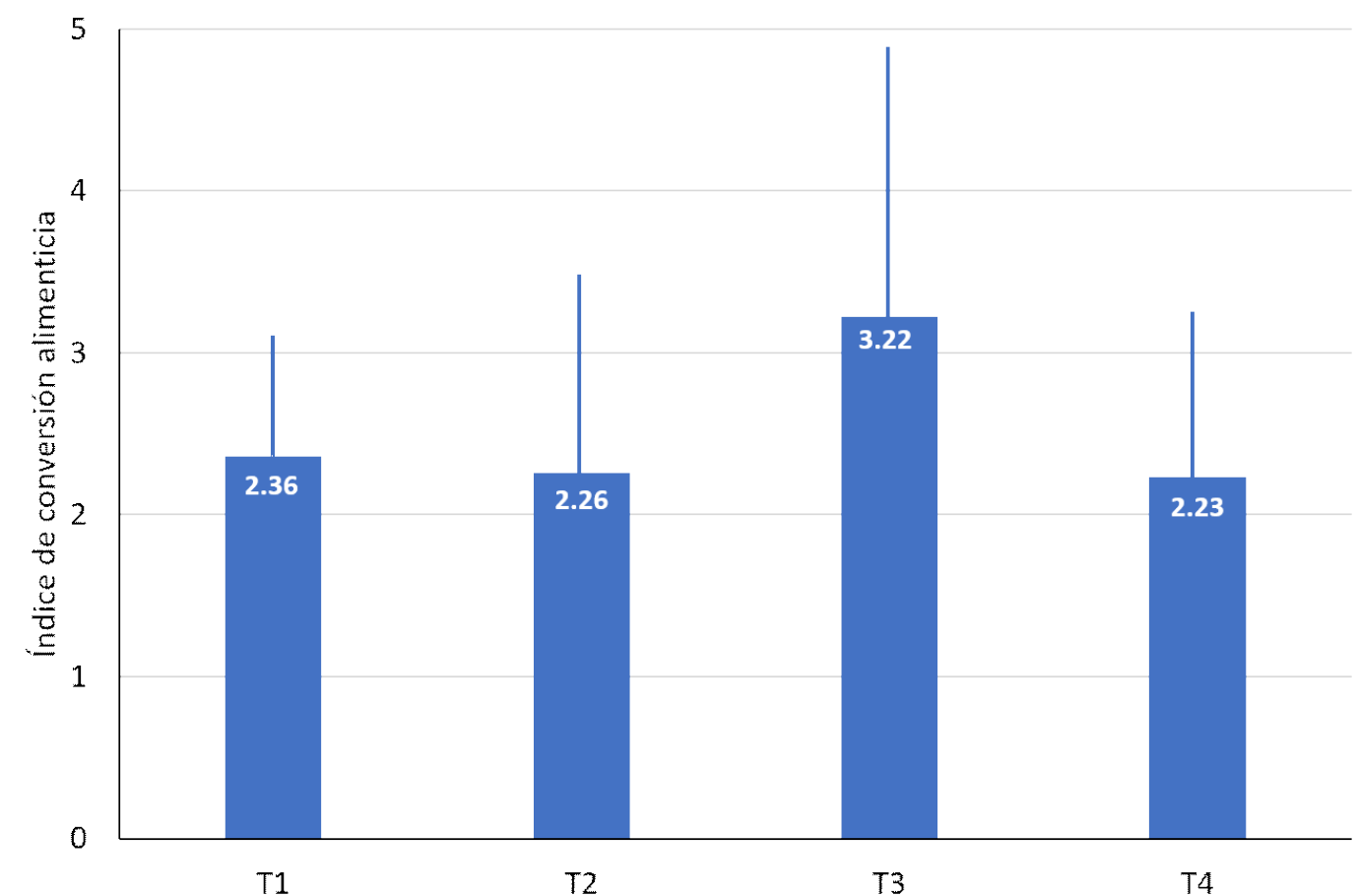

Figura 3. Índice de conversión alimenticia de cuyes de la raza Perú durante 40 días de recibir las dietas experimentales. T1: concentrado integral (control); T2: concentrado integral $+18 \%$ harina de tarwi; 33 : concentrado integral $+25 \%$ harina de mashua; T4: concentrado integral $+25 \%$ harina de mashua $+18 \%$ harina de tarwi 
rior en las dietas con tarwi, aunque sin diferencia significativa, posiblemente debido al menor contenido de carbohidratos (Chauca, 1997; Ojeda y Salazar, 2011), pero fue, en todos los casos, mayor a los reportes con residuos de cerveza (Cerna, 1997) y harina de alfalfa (Salcedo, 2017).

No hubo diferencias significativas en el ICA; sin embargo, se observó que los cuyes tuvieron un proceso de adaptación al alimento en las primeras semanas, siendo menor para el grupo alimentado con suplemento de mashua (T3, semana 3), posiblemente debido el menor contenido de fibra y mayor contenido de carbohidratos (Morales, 2009; Urresta, 2010). Los resultados alcanzados fueron menores a lo reportado con el uso de peletizados (Tarrillo et al., 2018) y dietas suplementadas con aceite de pescado y semilla de sacha inchi (Guevara et al., 2016).

El valor nutricional de la carcasa no mostró grandes diferencias entre tratamientos, aunque se registró un mayor contenido de grasa con la suplementación de mashua, efecto que se contrasta con otros resultados de adición de suplementos (Huamaní, 2015; Flores-Mancheno et al., 2017).

\section{Conclusiones}

- La suplementación con harina de tarwi tuvo un efecto positivo en la mejora de la respuesta de la ganancia de peso vivo, sin afectar significativamente el consumo de alimento y la conversión alimenticia.

- El suplemento con harina de mashua incrementó la proporción de grasa en las carcasas.

\section{Literatura Citada}

1. [AOAC] Association of Official Analytical Chemists. 1990. Official Methods for analysis of the AOAC. $15^{\text {th }}$ ed. Arlington, Virginia, USA: AOAC. 771 p.
2. Cerna MA. 1997. Evaluación de cuatro niveles de residuo de cervecería seco en el crecimiento - engorde de cuyes. Tesis de Ingeniero Zootecnista. Lima, Perú: Univ. Nacional Agraria la Molina. 84 p.

3. Chauca L. 1997. Producción de cuyes (Cavia porcellus). Estudio FAO producción y sanidad animal 138. Organización de las Naciones Unidas para la agricultura y alimentación. Roma: FAO. [Internet]. Disponible en: http://www.fao.org/3/w6562s/w6562s00.htm

4. Chauca L, Vega L, Valverde N. 2004. Evaluación del crecimiento de cuyes raza Perú alimentados con raciones con diferente densidad nutricional. En: XXVII Reunión de la Asociación Peruana de Producción Animal. Piura, Perú: APPA.

5. Collado K. 2016. Ganancia de peso en cuyes machos (Cavia porcellus), post destete de la raza Perú, con tres tipos de alimento - balanceado - mixta - testigo (alfalfa) en Abancay. Tesis de Ingeniero Agrónomo. Abancay, Perú: Univ. Tecnológica de Los Andes filial Andahuaylas. $92 \mathrm{p}$.

6. Cuibin $R$, Zea M, Palacios $P$, Norabuena M, Collazos P, Sotelo AM. 2020. Determinación de la digestibilidad y energía digestible de la harina de kudzu (Pueraria phaseoloides) en el cuy ( $\mathrm{Ca}$ via porcellus). Rev Inv Vet Perú 31: e19020. doi:10.15381/rivep.v31i4.19020

7. Flores-Mancheno CI, Duarte C, Salgado-Tello IP. 2017. Caracterización de la carne de cuy (Cavia porcellus) para utilizarla en la elaboración de un embutido fermentado. Revista Ciencia y Agricultura 14: 39-45.

8. Guevara V, Rojas M, Carcelén C, Bezada Q, Arbaiza F. 2016. Parámetros productivos de cuyes criados con dietas suplementadas con aceite de pescado y semillas de Sacha Inchi. Rev Inv Vet Perú 27: 715-721. doi:10.15381/rivep.v27i4.12560

9. Hidalgo L, Valerio HY. 2020. Digestibilidad y energía digestible y metabolizable del gluten de maíz, hominy feed y subproducto de trigo en cuyes (Cavia porcellus). Rev Inv Vet Perú 31: e17816. doi: 10.15381/rivep.v31i2.17816 
10. Huamaní Ñ, Zea M, Gutiérrez, R, Vilchez, P. 2016. Efecto de tres sistemas de alimentación sobre el comportamiento productivo y perfil de ácidos grasos de carcasa de cuyes (Cavia porcellus). Rev Inv Vet Perú 27: 486494. doi:10.15381/rivep.v27i3.12004

11. [INEI] Instituto Nacional de Estadística e Informática. 2018. Encuesta Nacional Agropecuaria 2017. Principales resultados. Pequeñas, medianas y grandes unidades agropecuarias. Lima: INEI. $123 \mathrm{p}$.

12. [INS] Instituto Nacional de Salud. 2017. Tablas peruana de composición de alimentos. Lima, Perú: Ministerio de Salud. $62 \mathrm{p}$.

13. León G, Silva S, Wilson C, Callacna C. 2016. Vitamina C protegida en concentrado de Cavia porcellus «cuy» en etapa de crecimiento-engorde, con exclusión de forraje. Sci Agropecu 7: 259-263. doi: 10.17268/sci.agropecu.2016.-03.14

14. [MINAGRI] Ministerio de Agricultura y Riego. 2019. Potencial del mercado internacional para la carne de cuy 2019. Lima, Perú: MINAGRI. 14 p.

15. [MINAGRI] Ministerio de Agricultura y Riego. 2018. Anuario estadístico de producción agrícola. Lima, Perú: Dirección General de Seguimiento y Evaluación de Políticas (DGESEP). 371 p.

16. Morales AG 2009. Evaluación de dos niveles de energía en el comportamiento productivo de cuyes de la raza Perú. Tesis de Médico Veterinario. Lima, Perú: Univ. Nacional Mayor de San Marcos. 51 p.

17. [NRC] National Research Council. 1995. Nutrient requirements of laboratory animals. $4^{\text {th }}$ Revised ed. Washington DC, USA: NRC. 192 p.

18. Ojeda LC, Salazar JA. 2011. Efecto de la suplementación con harina de chocho (Lupinus mutabilis Sweet) en el comportamiento productivo de cuyes $(\mathrm{Ca}$ via porcellus) en la fase de levante y engorde. Tesis de Ingeniero Zootec-nista. Pasto, Colombia: Univ. de Nariño. 27 p.
19. Perea D, Vergara V, Remigio R. 2007. Evaluación de la harina de yacón en dietas para cuyes en crecimiento. En: Resúmenes de alimentación en alimentación mixta e integral. Lima, Perú: Univ. Nacional Agraria La Molina. $82 \mathrm{p}$.

20. Quintana E, Jiménez, $R$, Carcelén F, San Martín F, Ara M. 2013. Efecto de dietas de alfalfa verde, harina de cebada y bloque mineral sobre la eficiencia productiva de cuyes. Rev Inv Vet Perú 24: 425-432. doi: 10.15381/rivep.v24i4.-2744

21. Reyes-Sánchez N, Vivas J, Aguilar J, Hernández J, Caldera N. 2018. Suplementación de cobayos (Cavia porcellus $\mathrm{L}$ ) con follajes fresco de morera (Morus alba) y moringa (Moringa oleifera). La Calera 18: 7-13. doi: 10.5377/calera.v18i30.7733

22. Rosales C, León F, Fajardo M, Jara $X$. 2014. Uso de suero de leche líquido en la alimentación de cuyes (Cavia porcellus) en la etapa de crecimiento y engorde. Maskana 5: 87-95. doi: 10.18537/mskn.05.02.08

23. Salcedo WA. 2017. Evaluación de harina de sangre bovina y harina de alfalfa (Medicago sativa) como fuentes de proteína en el alimento balanceado para cuyes (Cavia porcellus L). Tesis de Ingeniero Agroindustrial. Puno, Perú: Univ. Nacional del Altiplano. $77 \mathrm{p}$.

24. Salvatierra R, Rimac B, Chero O, Reyna W, Rosadio A, Maturrano $H$. 2018. Resistencia antimicrobiana y genotipificación de cepas de Salmonella Typhimurium aisladas de cuyes (Cavia porcellus) provenientes de granjas de producción intensiva de la ciudad de Lima, Perú. Rev Inv Vet Perú 29: 319327. doi: 10.15381/rivep.v29i1.14089

25. SánchezJ. 2019. Estudio de factibilidad para la producción y comercialización de la carne de cuy en el mercado arequipeño. Tesis de Ingeniero Industrial. Arequipa, Perú: Univ. Católica San Pablo. $31 \mathrm{p}$. 
26. Solorzano D, Vergara V, Remigio $R$. 2010. Evaluación de dos tipos de alimento balanceado con diferentes densidades nutricionales en la etapa de crecimiento en una crianza en jaulas. En: Resúmenes de alimentación en alimentación mixta e integral. Lima, Perú: Univ. Nacional Agraria La Molina. $82 \mathrm{p}$.

27. Sotelo M, Valenzuela R, Césare C, Alegría A, Norabuena M, Gonzáles $H$, Paitan A, Valderrama T, Echevarría R. 2020. Determinación de la digestibilidad y energía digestible del forraje seco de mucuna (Mucuna pruriens) en cuyes. Rev Inv Vet Perú 31: e17537. doi: 10.15381/rivep.v31i1.17537

28. Sullca JC. 2019. Suplementación mineral en cuyes primerizas en la etapa de gestación - Granja Agropecuaria de Yauris-UNCP, Huancayo - Región Junín. Tesis de Ingeniero Zootecnista. Junín, Perú: Univ. Nacional del Centro del Perú. 76 p.
29. Tarrillo E, Mirez P, Bernal M. 2018. Uso de alimento peletizado en crecimiento-engorde de cuyes mejorados (Cavia porcellus) en Chota. Rev Cienc No@ndina 1:94-103. doi: 10.37518/26636360X2020vln2p94

30. Tineo I. 2015. Evaluación de tres niveles de proteína en el engorde de cuyes mejorados en la EE, CANAÁN - INIA a $2750 \mathrm{msnm}$, Ayacucho. Tesis de IngenieroAgrónomo. Ayacucho, Perú: Univ. Nacional San Cristóbal de Huamanga. 57 p.

31. Urresta BO. 2010. Evaluación del valor nutricional de la harina de mashua (Tropaeolum tuberosum) en dietas para pollos de engorde. Tesis de Ingeniero Agroindustrial. Quito, Ecuador: Escuela Politécnica Nacional. $137 \mathrm{p}$.

32. Zambrano OE. 2015. Costos de producción de crianza artesanal y tecnológica del cuy (Cavia porcellus) en Cajamarca. Tesis de Maestría. Lima, Perú: Univ. Nacional Agraria la Molina. 81 p. 